\title{
EXPANSIÓN DE LA INDUSTRIA MEXICANA DE LA CERVEZA ¿DIVERSIFICAR A ÁFRICA?
}

\author{
Giovanni Granados Macías* \\ Cesaire Chiatchoua** \\ Omar Neme Castillo***
}

\section{Resumen}

El objetivo del documento es analizar la creciente demanda de cerveza en el continente africano como una estrategia de diversificación para las exportaciones de la industria mexicana. Se utilizó un análisis descriptivo y los resultados muestran que las ventajas de la entrada de México en el mercado africano son: el gran potencial del consumo, los crecientes niveles de ingreso, la demografía galopante, la expansión de la urbanización e industrialización y el crecimiento económico de los países entre otros factores. De las desventajas, se puede mencionar la fuerte corrupción, un marco legal deficiente y la falta de infraestructura. Finalmente, en un contexto de incertidumbre para la renegociación del Tratado de Libre Comercio de América del Norte, voltear hacia otros mercados parece una alternativa viable para México.

\section{Abstract}

The objective of the document is to demonstrate that the growing demand for commercial beer in Africa represents a diversification strategy for Mexican industry exports. Through a descriptive analysis, this work presents the panorama of the beer market in Africa on the one hand and on the

* Estudiante de la Licenciatura en Relaciones Internacionales, Facultad de Ciencias Políticas y Sociales. Universidad Nacional Autonoma de México.

** Profesor de Asignatura, Escuela Superior de Economía - Instituto Politecnico Nacional. chiatchoua@ yahoo.co.uk

***Profesor Investigador, Escuela Superior de Economía - Instituto Politecnico Nacional. oneme@ hotmail.com 
other hand the Mexican product offer. The results show the advantages of Mexico's entry into the African market like: The great potential of consumption, rising income levels, galloping demographics, the expansion of urbanization and industrialization, and the economic growth of countries. According to the disadvantages, we can mention the strong corruption, a deficient legal framework and the lack of infrastructure. Finally, at a time when the renegotiation of the Free Trade Agreement is fraught with uncertainty, it will be well for Mexico to turn to other markets.

Palabras clave: Mercado de cerveza, Mercados no tradicionales, México, África, exportaciones. KeyWords: Beer Market, Non-Traditional Markets, Mexico, Africa, Exports.

Clasificación JEL: O54 - O55

\section{Introducción}

En la última década, la industria de la cerveza se ha convertido en una de las más importantes para la economía mexicana, no sólo en términos del crecimiento del valor de la producción, sino por la generación de valor agregado y por lo niveles de inversión. De acuerdo con datos del Instituto Nacional de Estadística y Geografía (INEGI), entre 2009 y 2014, esta industria registró una tasa de cambio superior a la de otras ramas manufactureras como la de equipo de audio y de video, aparatos electrodomésticos, componentes electrónicos y de equipo de comunicación, estandartes del dinamismo exportador del país. Asimismo, en ese periodo el valor agregado muestra una tasa de crecimiento promedio anual de $4.6 \%$ por arriba de industrias como la refinación de petróleo, aparatos de línea blanca, motores y generadores eléctricos, maquinaria y equipo para la construcción, equipo de audio y video, radio y televisión.

Respecto a la producción de cerveza, se tiene que en el 2014 se alcanzaron los 8.95 mil millones de litros, con un crecimiento del 6\% respecto al 2013. Para posicionarse como el quinto mayor productor mundial de ese líquido con una cuota del $4.1 \%$; mientras tanto, las participaciones del mercado mundial de los líderes productores son 25\% de China, Estados Unidos con $11.5 \%$, Brasil con 7.1\% y Alemania con 4.8\%. La producción de cerveza para 2016 ascendió a 10.5 mil millones de litros, con un crecimiento de $8.1 \%$ respecto a 2015 para posicionarse como el cuarto lugar de productores de cerveza (milenio.com). No obstante, este sector realiza inversiones que le permiten incrementar su capacidad de producción. De acuerdo con Cerveceros de México, estas inversiones permitirán que el país escale posiciones en el ranking mundial. ${ }^{1}$ Así, se pronostica que para el 2020 la tasa de crecimiento alcance el $2.6 \%$ dado el mayor nivel de producción derivado de las nuevas plantas.

Grupo Modelo y Cuauhtémoc Moctezuma, los gigantes cerveceros con operaciones en el país, anunciaron inversiones cercanas a los 47 mil millones de pesos. 
Asimismo, las exportaciones mexicanas se colocaron como número uno en el mundo. En 2014 se exportaron 1.7 mil millones de litros con un valor de 1.6 mil millones de dólares. Para el 2015 el valor de lo exportado alcanzó los 2.5 mil millones de dólares, representando un notable crecimiento a tasa anual de 56\%. Esta dinámica exportadora le ha permitido desplazar a países tradicionalmente líderes en el sector, dada la calidad de su producto, como Holanda, Bélgica, Alemania, Estados Unidos e Inglaterra. Las cervezas mexicanas vendidas en el mundo representan el 19.9\%, esto es, prácticamente una de cada cinco cervezas consumidas internacionalmente es de origen mexicano.

La Secretaría de Economía señala que los principales destinos de las exportaciones de cerveza son Estados Unidos (72\%), Chile (5\%), Australia (5\%), Canadá (4\%), Reino Unido (2\%) (SE, 2017). En este sentido, en la actualidad, la cerveza producida en México se ha posicionado como el principal proveedor de cerveza de Australia, Argentina, Chile, Estados Unidos, Guatemala y Nueva Zelanda, el tercero de Canadá y el cuarto de China y Japón; no obstante, se vende en un total de 180 países.

A pesar del panorama positivo para el sector cervecero mexicano, se identifica el problema estructural común en las exportaciones del país: la concentración de ventas en un país. En este caso, casi 3 de cada 4 cervezas se exportan a Estados Unidos. Esto ha permitido que las cervezas mexicanas controlen el $60 \%$ del mercado estadounidense, seguidos por Holanda (17.7\%) y Bélgica (7.7\%).

La realidad de las exportaciones de cerveza mexicanas es la competencia en un mercado altamente peleado con crecimiento moderado. Una alternativa para incrementar las ventas al exterior es reorientar su oferta hacia otros mercados. En particular, la demanda de este producto por el continente africano ha crecido más que en cualquier otra región en los últimos cinco años con tasas cercanas al 5\%. ${ }^{2}$ Se espera que esta tasa se mantenga al menos hasta el 2020 , lo que llevaría a su consolidación como una verdadera opción de mercado al demandar de manera conjunta 3.7 millones de litros anualmente. La causa de este aumento en el consumo se debe al crecimiento de la población (el crecimiento promedio de la población africana en 2015 fue de $2.5 \%$, por arriba de la tasa mundial de $1.18 \%$ ), ${ }^{3}$ a procesos de urbanización (lo que es fundamental para incrementar sus niveles medio de ingreso) y al incremento del PIB per cápita (el PIB per cápita africano creció, en promedio, 5.9\% anualmente durante

2 En contraste, se pronostica que el crecimiento en los mercados maduros como el europeo o norteamericano será de $1 \%$ para el 2020.

3 Como ejemplo, se espera que para el 2050 la población de Nigeria sea la tercera más numerosa a nivel mundial y que la población en edad de trabajar africana supere la de China e India. Lo anterior sugiere que África vivirá un considerable aumento en el número de bebedores en edad legal para hacerlo y un aumento del mercado de consumo de 400 mil millones (globalriskinsights.com). 
2000-2015). ${ }^{4}$ Esta dinámica perfila a los países africanos como un mercado lucrativo para las exportaciones. Este incremento en la demanda resulta de especial interés para las cervezas artesanales. ${ }^{5}$ Se espera que el consumo de este tipo de cerveza aumente principalmente en las crecientes zonas urbanas y en aquellos lugares con mayor potencial para el turismo (beveragedaily.com).

Angola y Sudáfrica lideraron las importaciones de cerveza en 2015, el primero registró compras por 64.8 millones de dólares y el segundo por 64.7 millones. Detrás de éstos aparecen Guinea Ecuatorial, Mozambique, Congo, República Democrática del Congo, Argelia, Uganda, Zambia y Sierra Leona, todos con importaciones superiores a los 10 millones de dólares (worldrichestcountries.com). Además, Zambia, Etiopía, Costa de Marfil y Kenia muestran las mayores tasas de crecimiento de la región y se espera que para el 2020 estén entre los primeros diez mercados. Sudáfrica ocupa el primer lugar en el continente con 66.5 litros por persona al año, similar al consumo en México; seguido por Camerún con 31.9 litros, Kenia con 13.5 litros, Nigeria con 12.5, Uganda 11, Mozambique 10 y Tanzania 9 (worldfinance. com). Resulta claro que estas cantidades representan una pequeña proporción del consumo de los principales consumidores de cerveza globales. En 2014, los mayores niveles de consumo per cápita estuvieron liderados por República Checa con 143 litros al año, Alemania con 110 litros, Austria con 108 litros. Países como Estados Unidos, Inglaterra, Bélgica y Holanda observaron consumos que varían en un rango de 70 a 95 litros anuales por persona (euromonitor.com). De este modo, se distingue un amplio potencial del mercado africano para incrementar la demanda de este tipo de bebida alcohólica.

En concreto, el objetivo del documento es analizar la creciente demanda de cerveza comercial en los países del continente africano como una estrategia de diversificación para las exportaciones de la industria mexicana que, en los últimos años, ha aumentado su capacidad de producción afianzándose como uno de los líderes globales en la exportación de esta bebida.

Para ello, el documento se estructura, además de la introducción, en cuatro secciones. En la siguiente se sintetizan las ventajas teóricas de exportar y diversificar mercados; en la tercera, se describe la capacidad de oferta mexicana de cerveza; en la cuarta se resume el panorama del mercado africano de la cerveza. En la última sección se presentan los resultados respecto a las oportunidades de exportación-importación.

4 A pesar que la mayoría de la población africana es rural (62.1\%), las tasas de crecimiento urbano, de prácticamente $4 \%$ anual, son las más rápidas del mundo y casi duplican el promedio mundial (United Nations Population Division 2001). Se espera que estas tasas se estabilicen en un promedio de $3.5 \%$ anual durante los próximos 15 años, lo que significa que la proporción de la población urbana de África en el mundo aumentará del 10 al 17\% (United Nations Population Division 2001).

5 Dada la limitada regulación africana de bebidas artesanales "hechas en casa" existe la tendencia a percibirlas como riesgosas para la salud, provocando un cambio en las preferencias de los consumidores hacia cervezas más confiables, lo que abre un nicho de mercado para las cervezas artesanales de origen mexicano. 


\section{Teoría: ventajas de exportar y las ventajas de diversificar}

Si bien el mercado doméstico puede ser suficientemente desafiante dada la mayor oferta de cervezas extranjeras desde la intensificación comercial, participar en mercados globales mediante la diversificación de productos y de destinos representa una serie de ventajas en los niveles macro y microeconómico.

Ante un mercado doméstico con bajo crecimiento o en recesión, que conduce a una saturación del mismo por la mayor oferta y contracción de la demanda, se hace cada vez más difícil colocar el producto, por ende, las firmas pueden reorientarse a la exportación como una vía para mantener sus niveles de venta (Aulakh et al., 2000). Además, es posible ampliar los niveles de producción generando economías de escala principalmente en bienes de amplia aceptación en los mercados de destino (Malmberg et al., 2016), como la cerveza. El aumento en la producción reduce los costos unitarios e incrementa las ventas y rentabilidad. Las exportaciones de largo plazo pueden hacer que las empresas exportadoras amplíen sus instalaciones productivas apuntalando así la competitividad (Shave, 2011).

Asimismo, al exportar es posible emplear capacidad de producción ociosa, aumentando los niveles generales de eficiencia de los recursos existentes (Damijan y Kostevc, 2005). Como se venden más productos sin un aumento proporcional de los costos totales de producción, se reducen los costos unitarios favoreciendo así la productividad (Girma et al., 2004). Menores costos unitarios incrementan la competitividad de un bien en los distintos mercados y contribuye a la rentabilidad global del productor. Al respecto, las ventas externas consolidan los beneficios dado que los pedidos promedio de clientes internacionales tienden a ser de mayor tamaño que la de compradores domésticos como una forma de reducir los costos de logística (los pedidos son generalmente por contenedor y no por pallet), afectando las ventas totales y la rentabilidad total (Fryges y Wagner, 2010; Vu et al., 2014). En corto, la alta competencia establece como requisito para participar en el mercado global elevados estándares de eficiencia y calidad que deben alcanzar los exportadores no solo en el proceso productivo, sino en aspectos ambientales y culturales, a partir de las mejores prácticas internacionales (CE, 2012). También, al participar simultáneamente en distintos mercados, los márgenes pequeños derivados de precios bajos para competir en ciertos destinos se compensan con los márgenes mayores obtenidos en otros mercados (Wagner, 2014).

Acceder a mercados no tradicionales permite que las empresas, principalmente pequeñas y medianas, sobrevivan en tiempos económicos difíciles al diversificar su oferta por productos y por geografía y disminuir así su nivel total de riesgo ante las habituales variaciones del tipo de cambio (Agosin et al., 2012). ${ }^{6}$ Exportar a distintos mercados internacionales reduce

6 En la medida que más firmas se incorporen al proceso exportador penetrando barreras comerciales arancelarias o no, nuevos exportadores estarán en posibilidad de seguir ese mismo camino, ampliando en 
el riesgo por posibles fluctuaciones en el mercado local o de los actuales socios comerciales asociadas al ciclo económico, resultando en unos portafolios de demanda extranjera balanceado. De igual manera, diversificar hacia países del hemisferio sur contribuye a minimizar los efectos por fluctuaciones estacionales manteniendo una demanda equilibrada todo el año (Qiana y Lib, 1998).

En concreto, exportar contribuye al sistema económico a ampliar los niveles de producción $\mathrm{y}$, de esta manera, al crecimiento económico sustentable, al tiempo que impacta en los niveles de productividad (Romer, 1990) generando nuevo conocimiento, tecnología y habilidades en los productores nacionales (Husted y Melvin, 2007) y potenciando la innovación nacional (CE, 2012). También, la orientación exportadora permite colocar el superávit de oferta en diferentes economías favoreciendo una mayor captación de divisas que, a su vez, impactan positivamente en el equilibrio de la balanza de pagos.

Diversificar genera una redistribución del ingreso por exportaciones hacia ese nuevo sector apuntalando, a su vez, la actividad económica y el empleo en el país, al crear nuevas oportunidades laborales con mejores remuneraciones en el largo plazo, dada la mayor productividad de este factor (CE, 2012). En este sentido, las exportaciones se entienden como catalizadores del desarrollo y reductores de pobreza a través de mayores inversiones asociadas a las ventas externas (CE, 2012).

\section{Oferta cervecera}

La industria nacional de la cerveza está dominada por dos grandes grupos con una participación cercana al 98.9\% y que constituyen de facto un oligopolio. ${ }^{7}$ La reciente reconfiguración de esta industria es resultado de la venta de las dos principales firmas productoras Grupo Modelo a AB-Inbev y Cuauhtémoc-Moctezuma a Heineken, así como de la entrada a territorio nacional de Constellation Brands, la tercera cervecera más grande de Estados Unidos. A nivel mundial también se dan movimientos empresariales con efectos en la industria mexicana. En 2016 AB-Inbev compró SABMiller, en ese entonces segundo productor global, por una cantidad de 104 mil millones de dólares representando la mayor adquisición en la historia de la industria cervecera (bolsamexicanadevalores.com.mx). ${ }^{8}$

consecuencia la base de exportadores. Al respecto, contar con una base exportadora más extensa ayuda a reducir la inestabilidad exportadora a nivel agregado impulsando la inversión (Bleaney y Greenaway, 2001).

7 De acuerdo con Vargas (2013) el mercado de la cerveza mexicano muestra un índice de HerfindahlHirschman de 0.4378 , que se aproxima a una estructura de concentración oligopólica.

8 La cervecera resultante, con una capitalización bursátil superior a los 275 mil millones de dólares, se convertiría en una firma dominante no solo en la industria cervecera sino en el sector de bebidas ya que tendría la capacidad para adquirir a otros gigantes refresqueros como Coca-Cola o Pepsi (Forbes, 2015). 
La empresa belga-brasileña Ab-Inbev, que produce el 25\% de las cervezas del mercado global, alcanza una cuota del $55.9 \%$ en el mercado mexicano, mientras que la holandesa Heineken, tercer productor mundial, mantiene un peso del $43 \%$ en México. El resto del mercado es atendido por microcervecerías con un peso creciente de Constellation Brands. No obstante, se avizora una nueva etapa en la industria puesto que la resolución de la Comisión Federal de Competencia (CFC) restringe los contratos de exclusividad en la distribución de los productos de AB-Inbev y Heineken en establecimientos comerciales al 20\% de los puntos de venta (tiendas, bares, cantinas, etc.) (Expansión, 2013). ${ }^{9}$ En caso de incumplimiento podría sancionarse a la empresa infractora con hasta $8 \%$ de los ingresos anuales en México, representando bases para el crecimiento de pequeños productores. ${ }^{10}$

La industria mexicana de la cerveza tiene un papel de importancia creciente en la economía nacional que se refleja en aspectos como su contribución al PIB del $0.6 \%$ para un valor total superior a los 22 mil millones de dólares, aporta el 4\% de la recaudación total de impuestos (IEPS, IVA, ISR y nómina) y genera 55 mil empleos directos y 2.5 millones de indirectos (Mundo Ejecutivo, 2015). Asimismo, se estima que por cada 1.5\% de crecimiento en el mercado cervecero doméstico o $3 \%$ en las exportaciones, se generan un billón de pesos en inversión directa de la industria de la cerveza, se produce un millón de hectolitros de cerveza y se crean 3,500 nuevos empleos en agricultura (CISCM 2017).

La producción bruta total de cerveza es de alrededor de 78,403 millones de pesos (CE, 2014) que se genera en 55 unidades económicas. El valor de la producción cervecera equivale al $1.2 \%$ de la producción bruta total manufacturera, con una tendencia positiva en esta década, alcanzando 96.9 millones de hectolitros en 2015. El ritmo de crecimiento también es importante al registrar una tasa promedio anual de 3.9\% desde el 2010. Para el 2016 la producción fue de 105 millones de hectolitros (Expansión, 2017c). Esto le ha permitido posicionarse como una potencia productora mundial al ocupar el cuarto lugar, detrás de de China, Estados Unidos y Brasil, pero por arriba de países como Alemania, Holanda y Bélgica (beerectorio.mx).

La situación anterior se apuntala al considerar el segmento de productores artesanales. Actualmente, la industria agrupa más de 500 productores artesanales. El número de cerveceras

9 SABMiller en conjunto con otras cervecerías artesanales trataron de aumentar su presencia en el mercado mexicano pero dada la problemática para acceder a bares y restaurantes, parte de los canales de distribución de los gigantes cerveceros, solicitaron en 2013 un proceso de investigación por prácticas monopólicas en los mercados de distribución de cerveza (cervezaartsanalmexicana.mx).

10 De acuerdo con Expansión (2013), esta resolución permite, no obstante, que se ejerza un control duopólico por ambos grupos en el 20\% de los negocios, casi 100 mil tiendas para Modelo y 70 mil para CuauhtémocMoctezuma, además de excluir la venta en los canales modernos de tiendas de conveniencia, estadios, centros de entretenimientos. 
artesanales registra una tasa de crecimiento promedio de 38\% en los últimos cinco años. Según datos de la Acermex, cada 2.5 días en promedio se registra una apertura de una cervecería artesanal desde el 2010. Tan solo en 2016 se abrieron 144 cervecerías y el crecimiento estimado para ese año es de 62\% (Expansión, 2017). También, el buen desempeño de la industria mexicana se debe al aumento de la demanda, dado el creciente interés entre los consumidores por nuevas marcas comerciales y artesanales con diferentes sabores y consistencias (SRS, 2017). La favorable dinámica de la industria cervecera se refleja también en el sector externo con saldos superavitarios permanentes. Del 2010 al 2015 acumula 12,563 millones de dólares para un ritmo anual medio de cambio del $4.6 \%$. El saldo positivo se debe a que el volumen exportado ha crecido a un mayor ritmo (tasa anual promedio de $5.8 \%$ ), hasta alcanzar los 28.5 millones de hectolitros en 2015, aproximadamente $30 \%$ de la producción bruta total.

Para la Secretaría de Economía, el país ocupa la primera posición mundial como exportador de cerveza con presencia en más de 180 países (SE, 2017). Aún más, las principales marcas industriales y, en menor medida las artesanales, siguen estrategias de penetración de nuevos mercados tal como lo hiciera Corona algunas décadas atrás, llevando a que las marcas de cerveza mexicanas reporten el mayor crecimiento a nivel global, dado que son altamente consumibles y de moderado contenido alcohólico (mexiconewsdaily.com). Los principales destinos de la producción cervecera son Estados Unidos con el 80\% del total, Chile (5\%), Australia (5\%), Canadá (4\%) y Reino Unido (2\%). La capacidad de producción ha permitido que la cerveza de origen mexicano se posicione como el líder proveedor en los mercados de Estados Unidos, Australia, Chila, Guatemala, Argentina y Nueva Zelanda (mexico.mx). A pesar de la alta concentración de las ventas externas, la industria vislumbra una diversificación (mexiconewsdailay.com); por ejemplo, Reino Unido y China son el tercero y sexto consumidores más grandes del planeta con aumentos superiores al 50\% en 2016 frente al 2015. ${ }^{11}$

Adicionalmente, las cerveceras artesanales han accedido a mercados externos dado que cuentan con el nivel de madurez necesario para ello (El Financiero, 2017). Así, uno de cada diez litros de cerveza artesanal producida en México se envía a mercados internacionales. Asimismo, la capacidad exportadora de los productores artesanales se refleja en la participación en el mercado de Estados Unidos con 1.4\% del total. ${ }^{12}$ Además de exportar al vecino del norte, se ha iniciado un proceso de diversificación de destinos, reorientando parte de la producción a nuevos mercados como Centroamérica, Canadá, Australia, Nueva Zelanda, Francia, Bélgica y España, con la intención de aprovechar la depreciación actual del peso mexicano y el aumento en la demanda por productos tipo gourmet, de calidad e innovadores.

11 Otros países con importaciones desde México con crecimientos de doble dígito en 2016 son Australia, Canadá, Colombia, Bélgica e Irlanda (mexiconewsdailay.com).

12 Las empresas en el norte del país, principalmente Baja California, por su cercanía al mercado californiano, colocan aproximadamente la mitad de su producción en ese destino (El Financiero, 2017). 
En el 2016 el mercado superó los 71 millones de hectolitros de consumo en comparación al 2010 cuando se demandaron 65 millones, para un avance promedio anual de 1.4\% (beerectorio.mx). Esto ubica a los consumidores mexicanos en el puesto 39 al consumir 62 litros por persona al año. En estos términos, el mayor bebedor del mundo es la República Checa que consume 143 litros (lts), Alemania con 110 lts, Austria 108 lts, Estonia 104 lts, Polonia 100 lts, Irlanda con 93 lts, Rumania 90 lts, Lituania 89 lts, Croacia 82 lts y Bélgica 81 lts.

En el ranking de países por consumo total, se tiene que el mayor consumidor es China con el $23.5 \%$ del mercado total en 2015, seguido por Estados Unidos con 13.2\%, Brasil $5.1 \%$, Rusia $4.7 \%$, Alemania $4.6 \%$ y México con $4.0 \%$, por arriba de países como Reino Unido, Japón e India. ${ }^{13} \mathrm{Al}$ respecto, México es la segunda economía latinoamericana en el consumo de cerveza (kantarworldpanel.com). No obstante, el mercado mundial de consumo de cerveza se contrajo 1.8\% en 2016, la primera caída en los últimos 30 años (kirinholdings. co.jp), lo que se asocia al decrecimiento económico de tres grandes consumidores, China, Rusia y Brasil, donde el consumo de cerveza cayó más de 4\% dada la desaceleración de sus economías (thebeertimes.com).

Asimismo, con la primera cerveza artesanal en 1995 se ha ido formando una subcultura alrededor de esta bebida, principalmente en centros de consumo como la Ciudad de México, Tijuana, Guadalajara y Monterrey. Desde el 2009 se extiende a la población en general (Circulo Sonora, 2016), aunque con un consumo rezagado por el alto precio y difícil acceso a su consumo. La diferenciación de este tipo de bebidas, en alta calidad, sabores y marcas novedosas, ha generado un mercado especializado o gourmet que prefiere estas opciones a las cervezas industrializadas (Bernáldez, 2013), por lo que el mercado meta de la cerveza artesanal son personas entre 20 y 55 años y con un ingreso característico de la clase media alta. En pocas palabras, se enfocan en un nicho de mercado de consumidor gourmet interesado más en calidad que en cantidad (Circulo Sonora, 2016).

Por otro lado, adicional a los grandes consorcios con plantas productoras con capacidad global cercana a los 71 millones de hectolitros para AB-Inbev y 34 millones de hectolitros para Heineken en 2015, se distinguen tres tipos de cerveceras artesanales en función del tamaño de la producción. El 75\% de los productores tiene ventas de hasta 100 hectolitros (hl); 16\% registra un rango de 101 y hasta $500 \mathrm{hl}$; y $9 \%$ tiene ventas superiores a los $500 \mathrm{hl}$. En perspectiva con los grandes productores cerveceros, los artesanales producen un litro, mientras que el primer grupo fabrica 879 lts. También, por cada 975 litros que se consumen en el mercado solo un litro es de cerveza artesanal.

Entre productores industriales y artesanales, existen actualmente más de mil cervezas con nombre propio o marca agrupados en más de 92 estilos que se producen a lo largo de todas las

13 Si bien China demanda 54 mil millones de litros por año, el consumo per cápita es de apenas 4 litros (clarin.com). 
entidades federativas. Los grandes consorcios cerveceros operan entre los dos en 15 plantas con más de 28 marcas comerciales. Por un lado, AB-Inbev produce en Torreón, Ciudad de México, Guadalajara, Tuxtepec, Mazatlán, Ciudad Obregón, Zacatecas y Mérida. Por el otro, Heineken tiene sedes en Monterrey, Tecate, Navojoa, Guadalajara, Toluca, Orizaba y Meoqui. En contraste, los productores artesanales se concentran en Ciudad de México (15.9\%), Baja California (13.6\%), Jalisco (11.2\%) y Estado de México (7.7\%).

Por volumen de producción industrial destaca Zacatecas con el $17.7 \%$ de la producción bruta total, Coahuila con 14.6\%, Ciudad de México 12.4\%, Nuevo León 11.1\%, Oaxaca 10.8\% y el resto de entidades con $33.4 \%$. Para el caso artesanal, Jalisco contribuye con $34 \%$ de la producción nacional, seguida por Nuevo León (15\%) y Baja California ( $8 \%$ ). Los primeros cinco estados productores concentran el $71 \%$ del volumen procesado (beerectorio.com.mx).

Aparte, el costo promedio de producción de una cerveza industrial se ubica entre 12 y 15 pesos (Expansión, 2015). Simultáneamente, los fabricantes artesanales se caracterizan por altos costos de producción, con un promedio de 25 pesos (beerectorio.mx), lo que se asocia a tres factores principales: la capacidad instalada es reducida obstaculizando alcanzar economías de escala (El Financiero, 2014) y reflejándose en un costo promedio de hasta $120 \%$ mayor al de los grandes productores (Bernáldez, 2013); la escasez de materia prima (malta, levaduras o lúpulos) lleva a que se importe desde Estados Unidos o Europa, dado que la industria maltera nacional no cuenta con capacidad suficiente atender la demanda aparente (Bernáldez, 2013); los impuestos sobre el producto son $180 \%$ mayores frente al que paga la cerveza comercial, esto es, la cerveza artesanal paga al fisco al menos 10 pesos por litro, mientras que la industrializada alrededor de 3.5 pesos por litro (Milenio, 2016). El impuesto especial sobre producción y servicios (IEPS) grava a la cerveza con $26.5 \%$ sobre el precio de venta a lo que se le suma $16 \%$ por impuesto al valor agregado (IVA). En consecuencia, el precio de venta después de impuestos es aún mayor haciéndola menos competitiva contra la cerveza industrial. (Reforma, 2015). Lo anterior lleva a que el precio de venta de la cerveza artesanal varíe en un rango de entre 40 y 120 pesos, para un promedio de 60 pesos (Deloitte, 2017). ${ }^{14}$

Finalmente, las expectativas sobre el mercado mundial de la cerveza son optimistas ligados a la estrategia de expansión geográfica de las empresas cerveceras hacia nuevos puntos de venta. Esto se complementa por el creciente interés de los consumidores por probar diferentes marcas y descubrir nuevas oportunidades de consumo (euromonitor.com). En particular, se espera que la industria en México crezca por arriba de los 87 mil millones de pesos al finalizar el 2018 (SRS, 2017). Además, siguiendo a Euromonitor internacional, para el 2020 se incorporarán 5 millones de nuevos consumidores potenciales para ampliar el mercado a un total de 90 millones de personas con edad legal para beber (Rodríguez, 2016).

14 Una caja de 24 botellas de 335 mililitros tiene un precio final de 700 pesos, en comparación a 298 pesos para la cerveza industrial (Deloitte, 2017). 
Para responder a este crecimiento, existen planes de construcción de plantas productoras en el país que permitirán expandir la capacidad de oferta de los grandes consorcios cerveceros que actualmente se encuentra en el 97\% (aristeguinoticias.com)

Al respecto, Heineken invierte más de 7 mil millones de pesos para la construcción de una planta cervecera en Chihuahua desde el 2016 que procesaría 5 millones de hectolitros anuales, representando la inversión más importante para una planta en la historia de la empresa. Esta inversión se debe, en parte a que el mercado mexicano es el más grande de este grupo, con un 17\% del volumen total de producción (Rodríguez, 2016).

Al mismo tiempo, AB-Inbev inyecta 5 mil millones de pesos para la construcción de una fábrica de aluminio y una planta cervecera en Yucatán que permitirá aumentar la producción en 5 millones de hectolitros cada año. Se estima que la planta produzca en el mediano plazo 15 millones de hectolitros convirtiéndola en la segunda más grande del grupo después de la planta de Zacatecas, que genera entre 18 y 24 millones de hectolitros de cerveza anuales (Expansión, 2017a). Asimismo, se proyecta que 40\% de esa cantidad se exportaría.

El tercer competidor global en el mercado mexicano, Constellation Brands invierte poco más de 35 mil millones de pesos en Coahuila para una planta cervecera y una de envases que le permitirá producir hasta 25 millones de hectolitros por año (El Financiero, 2016), estableciendo la fábrica de cerveza más grande de Occidente incluidas todas las plantas de cerveza en Europa y Estados Unidos (El Financiero, 2017). Adicionalmente, llegó a un acuerdo para adquirir una planta del Grupo Modelo en Sonora por un valor de 600 millones de dólares con una capacidad de producción de 4 millones de hectolitros con la intención de abastecer los mercados de la costa Oeste de Estados Unidos (El Economista, 2016), en un marco en el que esta firma dispone de un contrato a perpetuidad con AB Inveb para el uso de todas las marcas de Grupo Modelo en territorio estadounidense (El Financiero, 2017).

En cuanto al segmento artesanal, también se tiene una perspectiva optimista puesto que las ventas registran cinco años consecutivos creciendo a tasas superiores al $40 \%$, pasando en 2012 de $15 \mathrm{mil} \mathrm{hl} \mathrm{a} 166 \mathrm{mil} \mathrm{hl} \mathrm{en} 2016$ y se proyecta un aumento del 59\% para 2017. A pesar del contexto económico internacional adverso, el escenario es favorable dada la alta calidad del producto artesanal, diferenciación de sabores y marcas y las nuevas estrategias empresariales orientadas a acceder a nuevos clientes de diferentes países (Milenio, 2016) como una alternativa más allá del duopolio que enfrentan en el mercado de origen.

Respecto a la incertidumbre generada por la renegociación del Tratado de Libre Comercio de América del Norte (TLCAN), que ha impulsado la exportación de esta bebida alcohólica a Estados Unidos, se espera continuar expandiendo la presencia en ese mercado; para ello, los productores artesanales deben enfocarse en producir una cerveza de buena calidad y cuidar los insumos utilizados en su producción (Milenio, 2016).

Finalmente, las dificultades que enfrentan los pequeños productores de cerveza artesanal principalmente en la distribución del producto final en el mercado nacional han limitado su 
expansión. No obstante, se vislumbra un camino para los fabricantes de mayor tamaño. Tanto AB-Inbev como Heineken han establecido alianzas estratégicas con marcas artesanales como Tijuana, Mexicali, Día de los Muertos, Bocanegra y Primus, entre otras -para ingresar a ese segmento- (Expansión, 2017b), implicando, entre otros aspectos, el acceso a canales de distribución y branding más amplios posibilitando así el desarrollo del mercado del segmento artesanal (Expansión, 2016).

\section{Demanda africana de cerveza}

La cerveza es la tercera bebida más consumida en el mundo, después del agua y el té; es también la bebida más compleja y variada. Puede tener sabor a humo, limón, café, chocolate, calafate, jengibre o cualquier otro sabor imaginable. La cerveza puede ser ácida y terrosa o puede ser amarga y aromática. El vino, principal rival de la cerveza, tiene muchas virtudes, pero no puede asemejarse en su variedad de sabores, aromas y texturas (Valencia, 2014). ${ }^{15}$

El consumo de cerveza ha aumentado de manera desigual en el mundo, Asia ha mantenido su ritmo de crecimiento durante más de 10 años, y el aumento de un 5,3\% en 2010 respecto al año anterior, esto se traduce en un porcentaje mayor (33,6\%) del mercado mundial en 2010 que en 2009, mientras el consumo de cerveza en Europa y América del Norte se redujo en un $2,4 \%$ y 1,4\%, respectivamente, de 2009 a 2010, el consumo en América Latina y África aumentó un $6,5 \%$ y $10,1 \%$, respectivamente.

El consumo mundial de cerveza fue impulsado por la fuerte demanda de los países en desarrollo, principalmente países de América Latina y África como Sudáfrica y Nigeria. (Cerveceros, 2014).

De hecho, en el ranking de los diez países líderes en la producción de cerveza en África para el 2015 se presenta: Sudáfrica y Nigeria respectivamente 32.1 y 27 millones de hectolitres, luego Angola con 11 millones, Camerún con 7.5 millones, Etiopía con 6.6 millones. Finalmente se posicionan Kenia con 4.74, la República Democrática del Congo con 4.5, Tanzania con 4.3, Zambia con 3.65 y Uganda con 3.27. ( africaprocessing, 2016).

SABMiller lidera el grupo, con participaciones en cuatro fábricas de África, Diageo y Castel poseen dos cada una y el Grupo O\&L poseen participaciones en una. Estas empresas representan el 80\% del mercado africano (Informe Sectorial de Mercados Potenciales en África, 2011).

Los tipos de cerveza son: Castel, Flag, la citada Gazelle, 33 Export, Star, Primus, Brakina, Bock, Kilimanjaro, Tusker, pero también variantes locales como la Guinnes o la Heineken son sólo algunas de las marcas más extendidas por África, donde el consumo de cervezas

15 Tomado de Oliver (2011). 
elaboradas tradicionalmente a partir del sorgo, mijo y otros cereales, (principalmente en zonas rurales), está siendo rápidamente sustituido por la amplia oferta mencionada anteriormente. (El país, 2017).

No se puede hablar de un precio único de la cerveza en África. La fijación de precios depende de varios factores de los cuales sobresalen: el lugar (bar, supermercado y restaurantes), la cantidad ( 25 centilitros, 33 centilitros u otra medida), la ciudad y el país. Para este estudio se considera el precio medio practicado en los países africanos, latinos y europeos. Se observa en la Tabla 1 que Etiopía es el país con el índice más bajo de precio de la cerveza, esto es inferior a 1 dólar, lo que no podría ser una oportunidad para México. Camerún es el país africano con el alto nivel de precio, lo que al contrario de Etiopía será conveniente para México invertir en ese lugar. Por otro lado Egipto, Angola y en cierta medida Sudáfrica son potenciales mercados para la cervecería mexicana porque presentan precios por arriba de México.

Tabla 1

Índice de Precio de la Cerveza 33 cl 2016 en dólares.

\begin{tabular}{cc}
\hline País & Precio medio de la cerveza \\
\hline Eslovaquía & 1.72 \\
Sudáfrica & 1.95 \\
México & 2.22 \\
Egipto & 2.49 \\
Guinea Ecuatorial & 1.78 \\
Nigeria & 2.12 \\
Camerún & 3.36 \\
Ghana & 2.17 \\
Angola & 2.53 \\
Kenia & 1.69 \\
Tanzania & 1.04 \\
Etiopía & 0.0058 \\
\hline
\end{tabular}

$\overline{\text { Fuente. Elaboración propia con base en proexca.es y goeuro.es. }}$ 


\section{Análisis de los Resultados}

En los últimos años, África ha sido considerada como una inversión "refugio" con un potencial ilimitado, calificada como una inversión de primer destino (Informe sectorial de mercados potenciales en África, 2011). Al respecto, las grandes empresas internacionales están apostando por un mercado africano de más de 14,106.03 millones de dólares anuales, el de mayor crecimiento de todo el mundo según el grupo de investigación cervecera Plato Logic, que pronostica un alza del 4.5\% en 2017 frente al rácano 1.4\% mundial (El País, 2017). Los factores de ventaja que presenta el mercado de la cerveza para la industria mexicana en África son numerosos, a saber:

i) Fortaleza del consumo, a pesar del efecto negativo que la crisis global tuvo y tendrá sobre el consumo en todo el mundo, especialmente en las economías más desarrolladas, la demanda en África se mueve en dirección opuesta a esa tendencia. Tomando en cuenta que el consumo per cápita es de 10.4 litros en ese continente, en comparación con los países más desarrollados, se aprecia un potencial de crecimiento significativo, a veces de más de ocho múltiplos. Aún más, esta previsión excluye a Sudáfrica, Botswana y Namibia, ya que estas economías han estimado niveles de consumo per cápita de más de 90 litros (Informe sectorial de mercados potenciales en África, 2011).

ii) Potencial demográfico, un factor clave es la dinámica demográfica, dónde una apabullante generación de jóvenes está llegando a la mayoría de edad y esto se traduce en un aumento de la demanda. En la última década la población del continente ha aumentado en 200 millones de personas y actualmente ya supera los mil millones de habitantes. Las proyecciones demográficas indican que el aumento será mayor en los próximos años, con una media del 2.2\% anual en el periodo 2010-2020. Mucho mayor que el 0.9\% que se proyecta para Asia. La ONU estima que el continente africano será responsable de más del 40\% del crecimiento de la población mundial hasta el año 2030. A este ritmo, en el año 2050 la población de África será de 2 mil millones de habitantes, superando a India (1,600 millones) y a China (1,400 millones). Para entonces, una de cada cinco personas en el mundo será africana (Unitedexplanations, 2013).

iii) Urbanización e industrialización, el crecimiento económico ha traído un desarrollo urbano importante en el continente africano. A manera de ejemplo, en 1960 había 50 millones de personas que habitaban en ciudades, en 2016 más de 500 millones de africanos viven en centros urbanos. Entonces el número de ciudades que superen el millón de habitantes será de 65, igualando a Europa y superando a Norteamérica que registra de 52 centros urbanos con esta caracterítica. Cabe señalar que con el $40 \%$ de su población viviendo en ciudades, África tiene una tasa de urbanización mayor que la India (30\%) y muy cercana a la de China (45\%) (unitedexplanations.org). El crecimiento de la clase media en Sudáfrica 
trae como consecuencia la urbanización del país, dos variables directamente relacionadas. Según datos del Banco Mundial (2015), la población urbana se estima en $64.8 \%$ de un total de 54.3 millones de habitantes y la tasa de urbanización anual en 1.59\%.

iv) Crecientes niveles de ingreso, de acuerdo con la Banca Africana de Desarrollo (BAD, 2011), entre 2000 y 2010, la clase media africana creció de manera considerable pasando de 205 millones de individuos (es decir $27 \%$ de la población africana) a 327 millones (34\% de la población). Estas cifras corresponden a una tasa de crecimiento de 59.6\%, tasa más elevada que la de población africana, que se sitúa a $27.6 \%$ en el mismo periodo (Fofana, 2015). Por otro lado, un estudio de la Standard Bank (2014) sobre la clase media de 11 países de África Subsahariana ${ }^{16}$ revela que entre 2000 y 2014, cerca de 6 millones de personas se incorporaron a la clase media para alcanzar un total de casi 8 millones de personas. Esta misma institución precisa que el número de vivienda perteneciendo a la clase media está destinada a triplicarse hasta alcanzar 22 millones de individuos al horizonte 2030, dado el contexto de crecimiento económico sostenido de la zona.

El crecimiento de los ingresos de la clase media en muchos países africanos es una realidad. Tomando el caso de Sudáfrica se observa una expansión del fenómeno. Según un estudio del Instituto de mercadotecnia estratégica, el ingreso de la clase media varía entre 1,550 y 4,800 dólares mensuales, lo que implica un aumento del poder adquisitivo y consecuentemente un aumento en el consumo. Los gastos de la clase media ascienden a 40 billones de dólares al año. (AfriqueRenouveau, 2013).

v) Estabilidad política, los años 90 en África fueron marcados por el cambio de sistema político al pasar de un sistema de partido único a un sistema multipartidismo que trajo consigo la democracia como factor fundamental para la estabilidad de los gobiernos. Esta situación permite establecer un clima de negocios favorable y atraer Inversión Extranjera Directa (IED). Vi) fuerte crecimiento económico durante 2001-2010. Por ejemplo, durante ese periodo Angola creció más que China o cualquier país en el mundo. En este mismo lapso se observa que de los 10 países de mayor crecimiento a nivel mundial, seis son africanos. Para el periodo 2011 a 2015, las proyecciones muestran que siete países de los 10 de mayor ritmo de crecimiento son africanos (unitedexplanations, 2013).

vii)Nivel de penetración escaso. África por la historia ha sido visto por una parte como un lugar de guerra, hambre, conflictos étnicos, peor aún con la llegada de la globalización los inversores voltearon hacia los países asiáticos. Por otra parte, África es un continente

16 Angola, Etiopía, Ghana, Kenia, Mozambique, Nigeria, Sudán del Sur, Sudán, Tanzania, Uganda y Zambia representan cerca de la mitad de la población africana y del PIB del continente. 
que ha sido controlado y sigue siéndolo pero en menor proporción por los países europeos, lo que ha complicado su apertura a los mercados internacionales. Después de los años 2000 y especialmente la crisis económica de 2009 ocurrida en los Estados Unidos ha dado un giro inesperado a África y su relación con el exterior. Muchas potencias económicas como los países asiáticos en la búsqueda de nuevos mercados han volteado hacia el continente negro y en la actualidad ha aumentado su presencia en este continente desplazando a los países europeos. Muy pocos lo saben pero según un estudio del Banco Africano de Desarrollo (2011), África presenta un progreso educativo con más niños en las escuelas, menos guerras civiles, un crecimiento sostenido, un mejor gobierno y una clase media creciente. Los países latinoamericanos tienen poca o nula presencia en este mercado.

Por otro lado, entre los principales obstáculos a la entrada de la cerveza mexicana al mercado africano aparecen: $i$ ) corrupción, fenómeno creciente en el continente que se ha establecido en un modo de vida, desde la alta administración pública de los países hasta la clase baja, pasando por las empresas y en las escuelas. Este fenómeno ha retrasado el desarrollo y crecimiento del continente, es un obstáculo para el desarrollo de los negocios, de hecho según un estudio de la consultora Maplecroft Global Risk Analytics realizado con la intervención de instituciones académicas internacionales y expertos específicos en cada materia, señala que seis de los diez países más corruptos del mundo están en África. (El mundo.es).

ii) Tramitología: Te Velde (2001) señala que el tiempo para establecer un nuevo negocio es entre uno y dos años en Uganda; en Ghana oscila entre 18 meses a tres años, en Tanzania y Mozambique, de seis meses a un año. En comparación con Asía, para establecer un negocio en Malasia, solo se tarda seis meses. Siguiendo el mismo orden de ideas, Asiedu (2004) afirma que en general, de los años ochenta a los noventa, la tasa de mejoras en la calidad institucional fue menor en los países del África Subsahariana que en otros países en desarrollo. Finalmente, la UNCTAD (1998) concluye que las regulaciones de IED que han liberalizado restricciones han contribuido significativamente a la mejora del clima de inversión. (Chiatchoua y Sibe, 2015).

iii) Infraestructura: en el crecimiento de las economías africanas aún no se ve reflejado el desarrollo de la infraestructura, esto ha obstaculizado la entrada de IED en el continente y las consecuencias de este déficit son visibles cuando uno recorre el continente y además afectan a todas las categorías sociales. Los ejemplos se dan en el transporte: viajar dentro de África es muy difícil debido a la falda de rutas viables lo que hace muy complicado, los desplazamientos entre ciudades y muchas veces los viajes se hacen eternos (Moya, 2014). 


\section{Conclusiones}

La industria de la cerveza se ha convertido en una de las más importantes para la economía mexicana, caracterizándose por su alta calidad e innovación reflejada en marcas y sabores diferenciados, de hecho, México es líder mundial en la exportación de la cerveza, donde la creciente demanda particularmente en zonas urbanas de la clase media africana representa una oportunidad histórica de acceso a nuevos mercados no tradicionales para el país.

Esta industria se divide en dos sectores principales, la cerveza industrial y la artesanal. En el segmento industrial, dos grupos AB-Inbev y Heineken concentran el 98.9\% de participación. La producción para el año 2016 alcanzó los 105 millones de hectolitros. En el ramo artesanal, la industria agrupa más de 500 productores con un volumen de exportación de 28.5 millones de hectolitros en 2015. México vende principalmente a los Estados Unidos, cerca de $80 \%$ de participación, esta concentración en un solo punto no es sostenible en un mundo competitivo donde México se ha visto rebasado en ese mercado por los productos chinos. Una diversificación de mercado representa una alternativa para México. Por otro lado, el crecimiento de los países africanos se ha visto reflejado en el consumo de cerveza. Sudáfrica, Botsuana y Namibia han estimado niveles de consumo per cápita de más de 90 litros, muy lejos del consumo per cápita que es de 10.4 litros en el continente. Factores como la aparición de una clase media, el fortalecimiento de los ingresos, el crecimiento de la urbanización, la entrada cada más de los flujos de IED y la democratización de los sistemas de gobierno pueden explicar los altos niveles de consumo de la cerveza en África; sin embargo, la corrupción, la falta de legislación clara y la deficiencia de la infraestructura pueden ser factores de freno de la entrada de México en el mercado de la cerveza africana.

Además, en relación a los ingresos de los consumidores en el continente africano, se puede afirmar que los consumidores africanos de menores ingresos ganan sólo 2 o 3 dólares al día. Pero en muchos países africanos, los ingresos per cápita están subiendo con rapidez, por lo que estos consumidores están convirtiéndose en blanco de las multinacionales, en momentos en que la población y el crecimiento se estancan en países más desarrollados fuera de África. (vanguardia.mx).

Finalmente, en un mundo altamente competitivo y de intensa innovación ya no es conveniente para México concentrar sus exportaciones en un solo país. Al respecto, el secretario de relaciones exteriores, Dr. Videgaray, señaló, en torno a la renegociación del Tratado de Libre Comercio, "México ampliará la relación comercial con América Latina, Europa y Asia", a estos se agrega África. El futuro se vislumbra incierto en las relaciones comerciales de México con sus principales socios, por lo que parece una oportunidad histórica de ampliar las relaciones comerciales con nuevos socios, en este contexto, África se presenta como una alternativa viable. 


\section{Referencias}

Agosin, M. Alvarez, R. y Bravo, C. (2012). "Determinants of export diversification around the world: 1926-2000", The World Economy 35(3):295-315.

Asiedu, E. (2004). "Policy reform and foreign direct investment in Africa: Absolute progress but relative decline", Development Policy Review 22 (1):41-48.

Aulakh, P., Rotate, M. y Teegen, H. (2000). "Export strategies and performance of firms from emerging economies: Evidence from Brazil, Chile and Mexico", Academy of Management 43(3):342-361.

Banca Africana de Desarrollo. (2011). "The middle of the pyramid: dynamics of the African.

Middle Class". En Fofana, A. (2015). "La llegada de una clase media africana. Un fenomeno todavia nuevo encore nouveau, Fuente de muchas oportunidades de negocios." BSI Economics.

Bernáldez, A. (2013). “Cerveza artesanal en México: ¿Soberanía cervecera y alimentaria?”, Culinaria, 6(2): 56-63.

Bleaney, M. y Greenaway, D. (2001). "The impact of terms of trade and real exchange rate volatility on investment and growth in sub-Saharan Africa", Journal of Development Economics 65(2):491-500.

Cadot, O., Carrére, C. y Strauss, V. (2011). "Export diversification: What's behind the hump?", Review of Economics and Statistics 93(2):590-605.

Chiatchoua, C. y Sibe, J. (2015). "Foreign direct investment in Sub-Saharan Africa (SSA): An opportunity for new developed countries (NDC)", Análisis Económico 73(30):8-12.

Damijan, J. y Kostevc, C. (2005). "Performance on exports: Continuous productivity improvements or capacity utilization", LICOS Discussion Papers 163/2005, LICOS Centre for Transition Economics.

Deloitte. (2017). La cerveza artesanal. Una experiencia multisensorial. Deloitte: México, DF.

Fofana, A. (2015). "La llegada de una clase media africana. Un fenomeno todavia nuevo encore nouveau, Fuente de muchas oportunidades de negocios." BSI Economics.

Fryges, H. y Wagner, J. (2010). "Exports and profitability: First evidence for German manufacturing firms", The World Economy 33(3):399-423.

Girma, S., Greenaway, A. y Kneller, R. (2004). "Does exporting increase productivity? A microeconometric analysis of matched firms", Review of International Economics 12(5):855-866.

Husted, S. y Melvin, M. (2007). International economics, Addison-Wesley: Boston.

Malmberg, A., Malmberg, B. y Lundequist, P. (2016). "Agglomeration and firm performance: Economies of scale, localization and urbanization among Swedish export firms", Environment and Planning A 32(2):305-321.

Qiana, G. y Lib, J. (1998). "Multinationality, global market diversification, and risk performance for the largest U.S. firms", Journal of International Management 4(2):149-170.

Oliver, G. (2011). The Oxford companion to beer. Oxford University Press: Nueva York.

Rendon, L, (2010). "Logro del crecimiento en el mercado global de la cerveza "South African Breweries" Estudio de caso.” Tesis de Maestría, Universidad Iberoamericana: México, DF.

Rodríguez, D. (2016). "El plan de Heineken para producir 500 millones de litros de cerveza en México", Alto Nivel. Disponible en: altonivel.com.mx/plan-heineken-producir-500-millones-litros-cervezaen-mexico.

Romer, P. (1990). “Endogenous technological change”, Journal of Political Economy 98(5):71-102.

Shave, J. (2011). "The benefits of geographical sales diversification: How exporting facilities capital investment", Strategic Management Journal 32(10):1046-1060.

Standard bank. (2014). "Understanding Africa's Middle Class". En Fofana, A. (2015). "La llegada de una clase media africana. Un fenomeno todavia nuevo encore nouveau, Fuente de muchas oportunidades de negocios." BSI Economics.

Te Velde, D. (2001), Policies towards foreign direct investment in developing countries: Emerging best practices and outstanding issues, Overseas Development Institute (ODI): Londres.

UNCTAD (1998), Trends and determinants. World Investment Report, United Nations: Nueva York. 
Valencia, O. (2014). "Rompiendo paradigmas en la experiencia del consumidor de cerveza en el punto de venta", Tesis de Maestría, Universidad de Chile, Departamento de Ingeniería Industrial.

Vargas, J. (2013). "Business strategy in Mexican beer industry: A case applying game theory", XVII Congreso Internacional en Ciencias Administrativas. Universidad del Valle de Atemajac.

Vu, H., Holmes, M., Lim, S. y Tran, T. (2014). "Exports and profitability: A note from quantile regression approach", Applied Economics Letters 21(6): 442-445.

Wagner, J. (2014). "Is export diversification good for profitability? First evidence for manufacturing enterprises in Germany", Journal of Applied Economics 46(33):4083-4090.

\section{Artículos de divulgación}

Africa Processing Food Solutions for Africa. (2016). "Le marche de la biere en Afrique", Disponible en: africaprocessing.com/fr/2016/11/01/le-marche-de-la-biere-en-afrique/

Círculo Sonora. (2016). Disponible en: circulosonora.com/2016/11/11/mercado-la-cerveza-artesanal/

El Economista. (2016). Disponible en: eleconomista.com.mx/industrias/2016/11/01/planta-grupomodelo-sera-constellation-brands

El Financiero. (2017). Disponible en: elfinanciero.com.mx/opinion/en-2017-piedras-negras-seracapital-cervecera-global.html

El Financiero. (2015). Disponible en: elfinanciero.com.mx/empresas/constellation-brands-alistaprimera-expansion-en-nava-para-finales-de-2015.html

El País. (2017). "África, el mercado de cerveza de mayor crecimiento". Disponible en: elpais.com/ internacional/2017/04/24/mundo_global/1493019619_390933.html

Expansión. (2016). Disponible en: expansion.mx/empresas/2016/08/12/subsidiaria-de-grupo-modelose-vuelve-titular-de-marcas-de-cerveza-artesanal

Expansión. (2017a). Disponible en: expansion.mx/empresas/2017/05/08/grupo-modelo-pone-enmarcha-su-planta-en-yucatan-con-una-inversion-de-8-500-mdp

Expansión. (2017b). Disponible en: expansion.mx/empresas/2017/02/13/la-sed-por-tener-una-cervezaartesanal-en-mexico.

Expansión (2017c). Disponible en: expansion.mx/empresas/2017/05/05/mexico-es-el-cuarto-productorde-cerveza-despues-de-china-eu-y-brasil

Forbes. (2015). Disponible en: forbes.com.mx/fusion-entre-ab-inbev-y-sabmiller-pone-peligro-asector-de-bebidas/

Herrera, K. y Jiménez, A. (2015). "Industria cervecera: Con sed de espumoso consumo", Mundo Ejecutivo. Disponible en: mundoejecutivo.com.mx/economia-negocios/2015/07/08/industriacervecera-sed-espumoso-consumo

Informe sectorial de mercados potenciales en África. (2011)." Bebidas alcohólicas. La cerveza", Disponible en: proexca.es/Portals/0/Documents/EstudiosMercado/Africa/2011.01.24_INFORME_ SECTORIAL_CERVEZA.Pdf

Milenio. (2016). Disponible en: milenio.com/negocios/cerveza_artesanal-acermex-Por_que_la_ cerveza_artesanal_es_tan_cara_0_722328003.html.

Moya, G, J, R. (2014). “Un acercamiento a las infraestructuras en África Subsahariana”, Disponible en www.africainfomarket.org/actualidad/informe/download/6529/17006/31

Organización Mundial de la Salud. (2014). "Rapport sur la consommation d'alcool et la santé dans le monde", Disponible en: maliactu.net/mali-rapport-2014-de-loms-sur-la-consommation-dalcoolun-rang-peu-flatteur-de-3e-mondial-sur-194-pays-pour-le-mali/

Radio France International Afrique. "Alcool: Quand les Africains boivent, ils boivent plus qu'ailleurs, Disponible en: rfi.fr/afrique/20141228-alcool-quand-africains-boivent-ils-boiventplus-ailleurs-oms

Reforma. (2015). Disponible en: negociosreforma.com/ 
SRS. (2017). Disponible en: relocationsrs.com.mx/beer-and-wine-industry-a-major-businessopportunity-in-mexico/

SE. (2017). Subsecretaría de Industria y Comercio, Secretaría de Economía. Disponible en: gob.mx/ se/artículos/industria-de-la-cerveza-en-México.

Unitedexplanations. (2013). El potencial económico de África: Las claves de su crecimiento. Disponible en: unitedexplanations.org/2013/07/09/el-potencial-economico-de-africa-las-clavesde-su-crecimiento/

Vanguardia.mx. (2013). “África, la última frontera de las cerveceras”. Disponible en: vanguardia.com. $\mathrm{mx} /$ africalaultimafronteradelascerveceras-1705454.html.

\section{Páginas internet consultadas}

aristeguinoticias.com/0411/kiosko/despunta-la-produccion-de-cerveza-industrial-y-artesanal-enmexico/

Banco Mundial. (2015). Población urbana (\% del total). Disponible en: datos.bancomundial.org/ indicador/SP.URB.TOTL.IN.ZS.

beveragedaily.com

bolsamexicanadevalores.com.mx/ab-inbev-compra-sabmiller/

cervezaartesanalmexicana.mx/cultura-cervecera/la-industria-cervecera-en-mexico

cerveceros.com. https://blog.birrapedia.com/consumo-de-cerveza-en-el-mundo/

clarin.com/viajes/ranking-paises-cerveza-consumo_0_SJ5KoTuvQx.html

elmundo.es/elmundo/2013/09/29/internacional/1380445411.html

euromonitor.com/beer-in-mexico/report

globalriskinsights.com

kantarworldpanel.com/mx/Noticias-/Mexico-2-lugar-en-consumo-de-Cerveza-en-Latinoamerica

kirinholdings.co.jp/english/news/2016/1221_01.html

mexiconewsdaily.com/news/mx-passes-germany-in-beer-production/

mexico.mx/es/articles/la-industria-de-la-cerveza-sube-como-la-espuma

thebeertimes.com/2017/06/06/consumo-mundial-cerveza-cayo-18-2016/ 